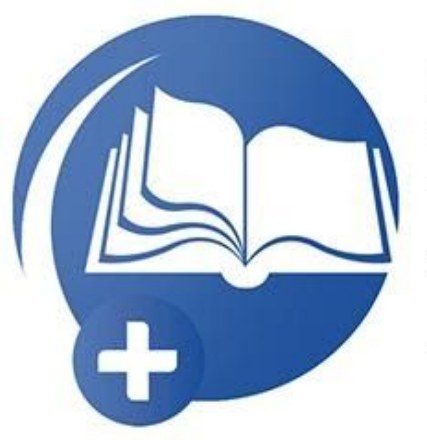

\title{
Preceptor modelo em nutrição clínica - percepção de estudantes e
}

\author{
preceptores
}

\author{
Fabrício da Paixão Gonçalves Pinto ${ }^{1}$ \\ Ida Helena Carvalho Francescantonio Menezes $^{2}$ \\ Alessandra Vitorino Naghettini ${ }^{3}$
}

\begin{abstract}
RESUMO
O preceptor em nutrição clínica atua como exemplo de conduta profissional para o estagiário no cenário da prática. Conhecer o perfil do preceptor contribuirá para uma melhor atuação desse profissional no aprendizado dos estudantes. O objetivo da pesquisa foi caracterizar os atributos do preceptor modelo em nutrição clínica sob a ótica de preceptores e estudantes. Foi realizado um estudo do tipo exploratório descritivo, de abordagem qualitativa, por meio da realização de entrevistas semiestruturadas. Participaram da pesquisa 12, sendo cinco de uma instituição hospitalar de ensino e sete da outra, e 15 estudantes. Dos 20 estudantes convidados a participar, cinco recusaram. Para a análise dos dados foi utilizada a análise de conteúdo de Bardin. Os resultados foram definidos em 3 eixos temáticos: Eixo 1, conceito, com categorias relacionadas ao ensino, ao cuidado e aquele se mostra como um espelho. Eixo 2, atributos que aproximam e eixo 3, atributos afastam do modelo, com categorias que apresentavam qualidades pessoais, profissionais e relacionadas ao ensino. As categorias revelaram uma percepção semelhante entre os dois grupos quanto aos atributos do preceptor modelo, no entanto apenas o grupo dos estudantes percebeu o conceito do preceptor como um espelho para o futuro profissional. $\mathrm{O}$ estudo aponta para a necessidade de consolidar o conceito do preceptor em nutrição clínica e aprofundar na percepção do preceptor visto como modelo. Tais informações podem auxiliar na formação da identidade profissional e no desenvolvimento do profissionalismo.
\end{abstract}

Palavras Chave: Preceptor. Estágio clínico. Ensino superior.

\section{Model preceptor in clinical nutrition - perception of students and preceptors}

\begin{abstract}
The preceptor in clinical nutrition acts as an example of professional conduct for the intern in the practice setting. Knowing the profile of the preceptor will contribute to a better performance of this professional in the students' learning. The objective of the research was to characterize the attributes of the model preceptor in clinical nutrition from the perspective of preceptors and students. A descriptive exploratory study with a qualitative approach was carried out through semi-structured interviews.

\footnotetext{
${ }^{1}$ Mestre em Ensino na Saúde pela Universidade Federal de Goiás, Goiânia, Brasil. E-mail: nutricionistafabricio@gmail.com.br

${ }^{2}$ Doutora em Ciências pela Universidade Federal de São Paulo, São Paulo, Brasil. Professora da Universidade Federal de Goiás, Goiânia. E-mail: idahelenamenezes@gmail.com

${ }^{3}$ Doutora em Medicina (Pediatria) pela Universidade Federal de São Paulo, São Paulo, Brasil. Professora da Universidade Federal de Goiás, Goiânia. E-mail: anaghettini@gmail.com
} 


\section{Programa de Pós-Graduação \\ em Ensino na Saúde - MEPES/UFG \\ DOSSIÊ \\ ENSINO NA SAÚDE}

V.17, N.01, 2021. ISSN: 1807-9342

Twelve participated in the research, five from a teaching hospital and seven from the other, and 15 students. Of the 20 students invited to participate, five refused. For data analysis, Bardin's content analysis was used. The results were defined in 3 thematic axes: Axis 1, concept, with categories related to teaching, care and that one is shown as a mirror. Axis 2, attributes that approach and axis 3, attributes move away from the model, with categories that had personal, professional and teaching-related qualities. The categories revealed a similar perception between the two groups regarding the attributes of the model tutor, however only the group of students perceived the concept of the tutor as a mirror for the professional future. The study points to the need to consolidate the concept of the preceptor in clinical nutrition and to deepen the perception of the preceptor seen as a model. Such information can assist in the formation of professional identity and in the development of professionalism.

Keywords: Preceptorship. Clinical Clerkship. Teaching.

\section{INTRODUÇÃO}

O preceptor é o profissional que atua no ambiente de trabalho e de formação, especificamente na área e no momento da prática clínica, junto ao estudante que será o futuro profissional (BOTTI; REGO, 2008). A função do preceptor é orientar o estudante nos diferentes cenários de prática. Portanto o trabalho de preceptoria exige do profissional o domínio de algumas habilidades e competências que se agrupam em quatro blocos de saberes: saber-conhecer, saber-fazer, saber-ser e saber conviver (MONTEIRO; LEHER; RIBEIRO, 2011).

Sob essa perspectiva a definição de um profissional percebido como um modelo, está no centro da formação do estudante. A excelência na prática é aprendida por meio da observação, experiência e reflexão crítica sobre a percepção do aluno no encontro clínico. Habilidades e conhecimentos são essenciais, mas colocá-los em prática no atendimento ao paciente é aprendido em interação individual com um modelo profissional (KENNY; MANN; MACLEOD, 2003).

Os debates acerca do processo de ensino e aprendizagem na área da saúde a respeito da formação profissional encontraram destaques nos seguintes assuntos: qualidade dos

\footnotetext{
${ }^{1}$ Mestre em Ensino na Saúde pela Universidade Federal de Goiás, Goiânia, Brasil. E-mail: nutricionistafabricio@gmail.com.br

${ }^{2}$ Doutora em Ciências pela Universidade Federal de São Paulo, São Paulo, Brasil. Professora da Universidade Federal de Goiás, Goiânia. E-mail: idahelenamenezes@gmail.com

${ }^{3}$ Doutora em Medicina (Pediatria) pela Universidade Federal de São Paulo, São Paulo, Brasil. Professora da Universidade Federal de Goiás, Goiânia. E-mail: anaghettini@gmail.com
} 


\section{Programa de Pós-Graduação \\ em Ensino na Saúde - MEPES/UFG \\ DOSSIÊ \\ ENSINO NA SAÚDE}

ITINERARIUS

REFLECIONIS

V.17, N.01, 2021. ISSN: 1807-9342

cuidados prestados em saúde, com atenção nas habilidades técnicas e científicas; a preocupação para importância das competências de caráter ético e humanístico, além de uma prática dedicada ao bem-estar dos pacientes acima dos interesses individuais (BERNAT, 2012). Assim, surge o termo profissionalismo que descreve um conjunto de valores, comportamentos e relações que sustentam a confiança que a sociedade tem nos profissionais, comprometidos com a integridade, o altruísmo, melhora contínua, excelência e trabalho em equipe (ROYAL COLLEGE OF PHYSICIANS OF LONDON, 2005).

Não obstante, a dificuldade que se coloca no processo de formação de novos profissionais se refere a maneira de ensinar/praticar/avaliar esse profissionalismo de forma responsável, priorizando princípios básicos da justiça. Espera-se uma formação onde o resultado seja um indivíduo com um ethos profissional que vincule as competências técnico-científicas à humana (LIE, 2009; MOSSOP, 2012). Nesse contexto, o preceptor é apresentado como um modelo para os estudantes e assume importância diante do fato de que as suas ações possam influenciar a formação e atuação do futuro profissional (HEALY et al., 2012). Em algumas áreas da saúde como medicina e enfermagem o modelo de preceptoria já é pesquisado (BOTTI; REGO, 2011; FRANKLIN, 2013; USHER et al., 1999; WRIGHT et al., 1998). No entanto, no curso de nutrição, especialmente na área clínica a literatura é escassa (MACLELLAN; LORDLY, 2008).

Nas Diretrizes Curriculares Nacionais (DCN) do Curso de Nutrição, documento orientador da proposta de projeto pedagógico dos cursos de nutrição do país, o estágio de Nutrição Clínica deve fazer parte da formação profissional, com a supervisão docente e acompanhamento de nutricionistas dos locais credenciados. O estágio deve ocorrer em ambientes que propiciem conhecimento e vivências, e deve estar relacionado com o processo saúde-doença, com o perfil epidemiológico e com as necessidades de saúde da população, proporcionando a integralidade das ações do cuidar em nutrição (BRASIL, 2001). A

\footnotetext{
${ }^{1}$ Mestre em Ensino na Saúde pela Universidade Federal de Goiás, Goiânia, Brasil. E-mail: nutricionistafabricio@gmail.com.br

${ }^{2}$ Doutora em Ciências pela Universidade Federal de São Paulo, São Paulo, Brasil. Professora da Universidade Federal de Goiás, Goiânia. E-mail: idahelenamenezes@gmail.com

${ }^{3}$ Doutora em Medicina (Pediatria) pela Universidade Federal de São Paulo, São Paulo, Brasil. Professora da Universidade Federal de Goiás, Goiânia. E-mail: anaghettini@gmail.com
} 


\section{Programa de Pós-Graduação \\ em Ensino na Saúde - MEPES/UFG \\ DOSSIÊ \\ ENSINO NA SAÚDE}

Resolução do Conselho Federal de Nutricionistas (CFN) 418/2008 cita a figura do Nutricionista orientador dentro do estágio curricular, definido como preceptor nesse trabalho, atuando em conjunto com a supervisão docente da instituição de ensino (CONSELHO FEDERAL DE NUTRICIONISTAS, 2008).

Dado a inexistência de uma definição clara do conceito do preceptor de nutrição clínica e seus papéis surgiu a ideia de realizar a presente pesquisa norteada pelos seguintes questionamentos: Qual o conceito de preceptor em Nutrição Clínica e quais os atributos do preceptor modelo nessa área sob a ótica de preceptores e estudantes? A realização desta pesquisa teve a finalidade de identificar o conceito de preceptor em Nutrição Clínica e caracterizar os atributos que aproximam do modelo desse profissional, sob a ótica de preceptores e estudantes.

\section{MATERIAIS E MÉTODOS}

Trata-se de estudo qualitativo de caráter exploratório, realizado no período de junho a julho de 2017, em dois hospitais escolas da região centro-oeste do Brasil.

Esse tipo de abordagem responde à questões específicas, as quais preocupam, nas ciências sociais, com um nível de realidade que não pode ser quantificado, pois se trabalha com um universo de significados, motivos, aspirações, dentre outros aspectos particulares do indivíduo, que corresponde a um espaço mais profundo das relações, que não podem ser demonstrados em números ou variáveis (MINAYO, 2003).

O presente estudo foi realizado durante a disciplina Estágio Supervisionado em Nutrição Clínica do curso de Nutrição de uma universidade pública da região centro-oeste do Brasil. As atividades da disciplina foram desenvolvidas em duas instiruições públicas, hospitalares de ensino.

\footnotetext{
${ }^{1}$ Mestre em Ensino na Saúde pela Universidade Federal de Goiás, Goiânia, Brasil. E-mail: nutricionistafabricio@gmail.com.br

${ }^{2}$ Doutora em Ciências pela Universidade Federal de São Paulo, São Paulo, Brasil. Professora da Universidade Federal de Goiás, Goiânia. E-mail: idahelenamenezes@gmail.com

${ }^{3}$ Doutora em Medicina (Pediatria) pela Universidade Federal de São Paulo, São Paulo, Brasil. Professora da Universidade Federal de Goiás, Goiânia. E-mail: anaghettini@gmail.com
} 


\section{Programa de Pós-Graduação \\ em Ensino na Saúde - MEPES/UFG \\ DOSSIÊ \\ ENSINO NA SAÚDE}

ITINERARIUS

REFLECIONIS

V.17, N.01, 2021. ISSN: 1807-9342

A coleta de dados foi realizada por meio de entrevista semiestruturada orientada por um roteiro de previamente elaborado pelo pesquisador e orientadores, com questões norteadoras que abordaram conceito de preceptor, atributos essenciais na prática da preceptoria que aproximam ou o afastam de ser identificado como um preceptor modelo.

Foi realizado um teste piloto com um público de características semelhantes aos participantes antes de aplicar o roteiro de entrevista da pesquisa. Após o teste, o questionário do estudante e do preceptor foram alterados, as perguntas eram idênticas para os dois grupos, portanto personalizamos mais o questionário de acordo com o perfil dos participantes. As respostas não foram utilizadas para o escopo de análise da pesquisa. Após esta fase o roteiro foi revisitado, feita nova análise e revisto para que as perguntas contemplassem os objetivos do estudo.

Foram considerados participantes da pesquisa nutricionistas da assistência que exerciam a função de preceptor em nutrição clínica com experiência de no mínimo seis meses, com atividades em enfermarias e ambulatórios. Em relação aos estudantes, aqueles regularmente matriculados na disciplina estágio supervisionado em nutrição clínica, ativos, do nono ou décimo período do curso de nutrição, matriculados no primeiro semestre de 2017 , ou ainda aqueles estudantes que já haviam cursado o estágio de Clínica no segundo semestre de 2016 e que no ano de 2017 cursavam o estágio de nutrição em saúde pública ou alimentação coletiva.

Foram excluídos do estudo estudantes com matrícula trancada, em licença maternidade/ou em exercício domiciliar e preceptores que estavam em férias, licença médica, nutricionistas com menos de seis meses de preceptoria ou aqueles que não exerciam a função de preceptoria na área da nutrição clínica.

A lista de nomes dos estudantes foi fornecida pela coordenação do curso, já as listas dos preceptores foram obtidas junto à coordenação da disciplina. Preceptores e alunos foram

\footnotetext{
${ }^{1}$ Mestre em Ensino na Saúde pela Universidade Federal de Goiás, Goiânia, Brasil. E-mail: nutricionistafabricio@gmail.com.br

${ }^{2}$ Doutora em Ciências pela Universidade Federal de São Paulo, São Paulo, Brasil. Professora da Universidade Federal de Goiás, Goiânia. E-mail: idahelenamenezes@gmail.com

${ }^{3}$ Doutora em Medicina (Pediatria) pela Universidade Federal de São Paulo, São Paulo, Brasil. Professora da Universidade Federal de Goiás, Goiânia. E-mail: anaghettini@gmail.com
} 


\section{Programa de Pós-Graduação \\ em Ensino na Saúde - MEPES/UFG \\ DOSSIÊ \\ ENSINO NA SAÚDE}

ITINERARIUS

REFLECIONIS

V.17, N.01, 2021. ISSN: 1807-9342

contatados por meio de e-mail ou telefone. As entrevistas foram realizadas em locais reservados, fechados e silenciosos, gravadas, com estudantes e preceptores e tiveram a duração média de 20 a 30 minutos.

Os dados foram analisados por meio da Análise Temática uma forma de análise de conteúdo a qual consiste em revelar os "núcleos de sentido" que compõem a comunicação. De forma operacional esse método se desdobra em 3 (três) fases. A primeira etapa chamada de pré-análise, uma fase de organização. A segunda etapa, que se baseia na exploração do material, o qual consiste em operações de codificação, decomposição ou enumeração. E a terceria etapa: tratamento dos resultados obtidos e interpretação (BARDIN, 2016).

Nesta pesquisa, após a descoberta das unidades temáticas, trabalhou-se com categorias pré-estabelecidas e subcategorias que emergiram, com isso foram recortadas 3 eixos temáticos com o propósito de responder aos objetivos:

- O eixo temático "Conceito" e suas respectivas categorias que surgiram após análise dos dados das entrevistas dos participantes, são elas: Profissional que ensina, Profissional que cuida do estudante e Profissional espelho;

- O eixo temático "Atributos que aproximam do modelo" e

- O eixo temático "Atributos que afastam do modelo".

Em relação aos dois últimos eixos temáticos foram consideradas categorias a priori de acordo com o referencial teórico, são elas: Qualidades relacionadas ao ensino, Qualidades pessoais, Qualidades profissionais, Dificuldades relacionadas ao ensino, Dificuldades pessoais e Dificuldades profissionais (LEEW et al., 2013; PAICE; HEARD; MOSS, 2002; WRIGHT; CARRESE, 2002).

O estudo foi submetido e aprovado como projeto pelo Comitê de Ética do Hospital das Clínicas das Universidade Federal de Goiás, sob o parecer de nº 2.055.893, em 2017, e seguiu

\footnotetext{
${ }^{1}$ Mestre em Ensino na Saúde pela Universidade Federal de Goiás, Goiânia, Brasil. E-mail: nutricionistafabricio@gmail.com.br ${ }^{2}$ Doutora em Ciências pela Universidade Federal de São Paulo, São Paulo, Brasil. Professora da Universidade Federal de Goiás, Goiânia. E-mail: idahelenamenezes@gmail.com

${ }^{3}$ Doutora em Medicina (Pediatria) pela Universidade Federal de São Paulo, São Paulo, Brasil. Professora da Universidade Federal de Goiás, Goiânia. E-mail: anaghettini@gmail.com
} 


\section{Programa de Pós-Graduação \\ em Ensino na Saúde - MEPES/UFG \\ DOSSIÊ \\ ENSINO NA SAÚdE}

ITINERARIUS

REFLECIONIS

V.17, N.01, 2021. ISSN: 1807-9342

as normas estabelecidas pela Resolução n466, de 12 de dezembro de 2012 (BRASIL, 2012).

Os entrevistados assinaram Termo de Consentimento Livre Esclarecido.

\section{RESULTADOS E DISCUSSÃO}

A pesquisa possibilitou traçar o perfil dos entrevistados composto na sua totalidade por mulheres. No grupo de preceptores o tempo de atuação na área da preceptoria foi predominantemente pequeno, cerca de dois a três anos e a maioria não possuía prática docente.

Foram abordadas $12(100 \%)$ preceptoras, sendo cinco de uma instituição hospitalar de ensino e sete da outra, e 20 (100\%) estudantes, destes 5 (25\%) recusaram a participar.

A análise das falas das preceptoras e estudantes nas entrevistas identificou a semelhança em dois eixos temáticos. Do primeiro eixo, denominado Conceito, emergiram as categorias profissional que ensina e profissional que cuida do estudante. No grupo (estudantes) emergiu mais uma categoria sendo essa profissional espelho. O segundo eixo temático, Atributos que aproximam do modelo, foi composto pelas categorias qualidades relacionadas ao ensino, qualidades pessoais e qualidades profissionais. Diante da semelhança de percepções optou-se por uma análise conjunta dos dados, com exceção da categoria especifica que emergiu no grupo de estudantes. O terceiro eixo foi definido a partir de categorias referentes a atributos que afastam do modelo, representados por dificuldades pessoais, profissionais e relacionadas ao ensino.

\subsection{Conceito}

Profissional que ensina

\footnotetext{
${ }^{1}$ Mestre em Ensino na Saúde pela Universidade Federal de Goiás, Goiânia, Brasil. E-mail: nutricionistafabricio@gmail.com.br ${ }^{2}$ Doutora em Ciências pela Universidade Federal de São Paulo, São Paulo, Brasil. Professora da Universidade Federal de Goiás, Goiânia. E-mail: idahelenamenezes@gmail.com

${ }^{3}$ Doutora em Medicina (Pediatria) pela Universidade Federal de São Paulo, São Paulo, Brasil. Professora da Universidade Federal de Goiás, Goiânia. E-mail: anaghettini@gmail.com
} 
Essa categoria no grupo dos preceptores agrupou a subcategoria Profissional que orienta/ensina o aluno nas questões práticas e teóricas. O preceptor além da orientação teórica, ele deverá orientar conteúdos práticos.

[...] vai supervisionar a prática dele ali orientando, ensinando as rotinas do serviço, as diferenças, às vezes do que que é teórico do que que é prático, como aplicar o que ele aprendeu no dia a dia do serviço. (P3)

Nestas transcrições das falas dos preceptores é demonstrado que o profissional preceptor utiliza da prática para o ensino aos estudantes. Para tanto, recorre ao conhecimento teórico adquirido no decorrer do curso e o utiliza na rotina de trabalho para repassá-lo ao futuro nutricionista. O preceptor tem papel fundamental de integrar os conceitos e valores da escola e do trabalho (BOTTI; REGO, 2008).

Do grupo de estudantes emergiu a subcategoria: um profissional que está no estágio acompanhando a rotina e a prática exercida, essa subcategoria é idêntica a subcategoria anterior emergida do grupo de preceptores.

[...] É você dar aporte para o estudante que está lá, de todas as formas, não só na hora que ele estiver atendendo, mas na parte teórica ou até na parte emocional [...] mostrar como que faz [...] é ir junto e fazer junto. (A2)

Dentre as principais atribuições do preceptor, o papel de orientar, dar suporte, compartilhar e ampliar saberes teórico-práticos entre os estudantes tem destaque. O preceptor deve auxiliar os graduandos na adaptação da nova profissão possibilitando aos estudantes a aprendizagem pela percepção da realidade concreta (CASSOLA et al., 2014). O acompanhamento do preceptor durante o estágio é fundamental para a descoberta em conjunto de soluções para problemas reais, auxiliando o aluno em suas dúvidas sobre teoria e prática.

\footnotetext{
${ }^{1}$ Mestre em Ensino na Saúde pela Universidade Federal de Goiás, Goiânia, Brasil. E-mail: nutricionistafabricio@gmail.com.br ${ }^{2}$ Doutora em Ciências pela Universidade Federal de São Paulo, São Paulo, Brasil. Professora da Universidade Federal de Goiás, Goiânia. E-mail: idahelenamenezes@gmail.com

${ }^{3}$ Doutora em Medicina (Pediatria) pela Universidade Federal de São Paulo, São Paulo, Brasil. Professora da Universidade Federal de Goiás, Goiânia. E-mail: anaghettini@gmail.com
} 


\section{Programa de Pós-Graduação \\ em Ensino na Saúde - MEPES/UFG \\ DOSSIÊ \\ ENSINO NA SAÚDE}

Profissional que cuida do estudante

Dessa categoria no grupo de preceptores emergiu a subcategoria acolhimento no serviço. O preceptor deve auxiliar e acompanhar o novo estudante no campo da prática.

[...] receber esse aluno, acolher e orientar o aluno no sentido de quais são as demandas do serviço... eu acompanhar e auxiliar de alguma maneira na formação desse aluno... esse acolhimento é mais essa questão de passar uma tranquilidade também, uma certa segurança para o aluno, que ele não tem inicialmente. (P9)

Em um estudo realizado por Feuerwerker (2011), sobre a formação prática dos médicos, verificou-se que a apresentação ao estudante do processo de funcionamento na unidade de saúde é uma função mediada pelo preceptor. Ou seja, é papel deste profissional, propor, viabilizar e problematizar atividades que sejam relevantes para à produção do perfil profissional, de tal forma que faça sentido para estudantes, unidade e usuários. No entanto na área da nutrição é necessário um esforço ainda maior, pois ainda não foi consolidado o conceito de preceptor.

Já na fala dos estudantes emergiu uma subcategoria diferente do grupo dos preceptores: Oferece suporte.

[...] é dar o suporte para o estudante, para o estagiário no sentido de mostrar os caminhos, em primeiro momento e depois deixar ele seguir. Sempre com essa orientação prévia [...] (A15)

Conforme Franco, Montes e Silva (2013), em pesquisa onde estudantes de Medicina relataram sua visão sobre o papel da preceptoria, eles deram ênfase ao desejo em executar a função do estágio ao lado do preceptor. Sentimento de segurança transmitido pela proximidade, disponibilidade e paciência para esclarecimento de dúvidas e o apoio na realização de procedimentos, são aspectos que conferem atenção e apoio aos estudantes. Assim, observa-se a notória importância da presença do preceptor durante o estágio, no

\footnotetext{
${ }^{1}$ Mestre em Ensino na Saúde pela Universidade Federal de Goiás, Goiânia, Brasil. E-mail: nutricionistafabricio@gmail.com.br

${ }^{2}$ Doutora em Ciências pela Universidade Federal de São Paulo, São Paulo, Brasil. Professora da Universidade Federal de Goiás, Goiânia. E-mail: idahelenamenezes@gmail.com

${ }^{3}$ Doutora em Medicina (Pediatria) pela Universidade Federal de São Paulo, São Paulo, Brasil. Professora da Universidade Federal de Goiás, Goiânia. E-mail: anaghettini@gmail.com
} 


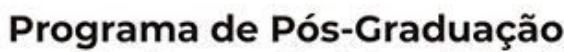

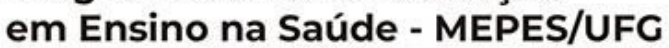 \\ DOSSIÊ \\ ENSINO NA SAÚDE}

V.17, N.01, 2021. ISSN: 1807-9342

auxílio da rotina do dia onde surgem várias dúvidas de conduta e também na orientação de protocolos clínicos.

Profissional espelho

Essa categoria emergiu do grupo dos estudantes, e mostra a importância do exemplo do preceptor no campo da prática. Esse profissional é visto como elemento fundamental na formação do estudante.

[...] ele também um elemento fundamental na construção de parte daquilo que você é como profissional, querendo ou não ele influencia muito isso, tanto na forma como ele trabalha - o preceptor, querendo ou não, é um espelho. Ele pode ser um espelho tanto para eu não me inspirar, como pode ser uma pessoa que me inspiro diante da sua prática no serviço. Se eu vejo que o preceptor é um bom profissional, vou querer ser como ele $[\ldots](\mathrm{A} 12)$

A ideia de profissional espelho reforça o papel de preceptor descrito como aquele que é o orientador. As falas também vão ao encontro da pesquisa realizada, que definiu o papel do preceptor como: orientador, exemplo, supervisor, tutor, guia, pai, amigo, professor, moderador, facilitador, parceiro e educador (BOTTI; REGGO, 2011).

Nota-se que dentro do grupo de preceptores não surgiram categorias com esta temática. Pode ser porque este grupo não percebe sua função como modelo no campo de estágio. Entretanto, para a modelagem de papéis ser mais eficaz, segundo Bandura (2008), esse processo deve ocorrer de forma intencional, ou seja, o preceptor deve conhecer este papel no processo de ensino aprendizagem.

\subsection{Atributos que aproximam do modelo}

Qualidades Relacionadas ao Ensino

\footnotetext{
${ }^{1}$ Mestre em Ensino na Saúde pela Universidade Federal de Goiás, Goiânia, Brasil. E-mail: nutricionistafabricio@gmail.com.br ${ }^{2}$ Doutora em Ciências pela Universidade Federal de São Paulo, São Paulo, Brasil. Professora da Universidade Federal de Goiás, Goiânia. E-mail: idahelenamenezes@gmail.com

${ }^{3}$ Doutora em Medicina (Pediatria) pela Universidade Federal de São Paulo, São Paulo, Brasil. Professora da Universidade Federal de Goiás, Goiânia. E-mail: anaghettini@gmail.com
} 


\section{Programa de Pós-Graduação \\ em Ensino na Saúde - MEPES/UFG \\ DOSSIÊ \\ ENSINO NA SAÚDE}

V.17, N.01, 2021. ISSN: 1807-9342

As preceptoras relataram a importância de o profissional ser comprometido com o estudante, sentir responsável pelo processo de aprendizado. Além do mais, deve ocorrer uma atitude ativa dos preceptores com intenção de proporcionar experiências da prática aos estudantes.

[...] acho que o mais importante também é se sentir responsável por aquele estagiário, entende, responsável no sentido de que estar participando do aprendizado... (P5)

Missaka e Ribeiro (2011) destacam que o preceptor tem papel fundamental na formação médica e demais áreas da saúde, ao integrar a teoria e a prática no contexto da assistência. As experiências vividas no campo da prática é um diferencial que o preceptor pode oferecer para o aluno. Segundo as falas das preceptoras quando o estudante participa das atividades do hospital, esta vivência pode acrescentar habilidades e conhecimento no aprendizado da profissão do nutricionista clínico. Portanto, no exercício da preceptoria não existe um ator principal, o processo é direcionado em conjunto: preceptor, estudantes e usuários do serviço, que devem estar abertos a compartilhar suas experiências (CERQUEIRA, 2011).

O Feedback efetivo foi outro atributo apontado pelo grupo dos preceptores:

[...] eu acho que chamar a atenção da forma mais tranquila, quando tem que chamar a atenção, mas ao mesmo tempo ser firme [...] (P3)

Conforme Borges e colaboradores (2014), o feedback efetivo é uma das estratégias educacionais e avaliativas com grande evidência de eficácia na educação das profíssões em saúde. Preceptores desses cursos precisam ser treinados para prover feedback efetivo. De acordo com a fala acima o preceptor deve ter habilidade para prover feedback para os estudantes. Em pesquisa realizada por Young e colaboradores (2014) foram identificados

\footnotetext{
${ }^{1}$ Mestre em Ensino na Saúde pela Universidade Federal de Goiás, Goiânia, Brasil. E-mail: nutricionistafabricio@gmail.com.br

${ }^{2}$ Doutora em Ciências pela Universidade Federal de São Paulo, São Paulo, Brasil. Professora da Universidade Federal de Goiás, Goiânia. E-mail: idahelenamenezes@gmail.com

${ }^{3}$ Doutora em Medicina (Pediatria) pela Universidade Federal de São Paulo, São Paulo, Brasil. Professora da Universidade Federal de Goiás, Goiânia. E-mail: anaghettini@gmail.com
} 
alguns fatores associados à excelência de preceptores de farmácia, tais como fornecer feedback, acessibilidade e discussão encorajada.

Para os estudantes, além da forma como ocorre o processo de ensino aprendizagem, também foi mencionado o respeito ao tempo do estudante:

[...] que tenha essa forma de passar o conteúdo, que tenha paciência também, porque isso é uma das coisas que sentimos muita falta. As vezes não tem a paciência de explicar, porque temos as dúvidas estupidas as vezes e por conta de receio de não responder, então, tem que ter paciência, tem que orientar a forma correta de passar uma dieta, de prescrever [...] (A15)

Para Autonomo e colaboradores (2015) a rotina do preceptor junto aos estudantes traz mudanças no cotidiano de trabalho, pois exige tempo e atenção para a sua formação. A falta de paciência de alguns preceptores pode gerar um certo desconforto para o estudante no momento que ele tem dúvidas, talvez essa impaciência seja fruto de uma rotina cheia, onde o preceptor divide atividades de assistência e ensino.

Autonomia no campo da prática, foi outro atributo que emergiu das falas dos estudantes:

[...] deixando o estagiário também seguir com suas próprias pernas a partir de um determinado momento [...] deixar ele seguir é a partir do momento que você já deu todas as informações e você percebe que ele já está com tudo o que ele precisa para ele continuar seguindo, é, tipo assim, observar mais de longe [...] (A15)

Conforme Ramos (2010), ao revisitar a Pedagogia da Autonomia de Freire, para que o processo educativo se concretize, o estudante precisa de espaço livre para desenvolver ou experimentar a curiosidade, que o auxiliará na trajetória que irá percorrer da ingenuidade à criticidade. Neste sentido o educador tem papel crucial neste processo, já que o espaço aberto

\footnotetext{
${ }^{1}$ Mestre em Ensino na Saúde pela Universidade Federal de Goiás, Goiânia, Brasil. E-mail: nutricionistafabricio@gmail.com.br ${ }^{2}$ Doutora em Ciências pela Universidade Federal de São Paulo, São Paulo, Brasil. Professora da Universidade Federal de Goiás, Goiânia. E-mail: idahelenamenezes@gmail.com

${ }^{3}$ Doutora em Medicina (Pediatria) pela Universidade Federal de São Paulo, São Paulo, Brasil. Professora da Universidade Federal de Goiás, Goiânia. E-mail: anaghettini@gmail.com
} 
cabe a ele propiciar, entendendo que educar não se trata de transferir conhecimento e que educar e educando, se completam.

Por último, a fala dos estudantes revelou o seguinte atributo: Habilidade em ensinar.

[...] Porque estamos aqui para aprender, então se a pessoa não sabe explicar você vai sair do estágio da mesma forma... Então para mim, tem que saber passar o conhecimento, mesmo que seja para passar norteando-o. Porque as vezes não é passar tudo que você sabe, mas pelo menos direcionar... de passar o que deve ser feito... sanar minhas dúvidas no decorrer do meu serviço e no decorrer da minha prática, e me apresentar como que tem que ser feito [...] (A7).

Neste estudo, do total de preceptores entrevistados, nove nutricionistas (75\%) não tinham experiência em docência. A habilidade em ensinar é um problema na formação do preceptor, pois de maneira geral nos cursos da área da saúde não se aprende a ser professor. Tradicionalmente os cursos da área da saúde se esquecem que, além da competência técnica, são necessárias habilidades concernentes ao âmbito pedagógico, para que seja possível compreender, planejar e executar ações educativas (MOHR, 2011). É necessário que os cursos de nutrição em conjunto com os serviços de saúde promovam cursos de formação continuada para os preceptores, abordando sobretudo temas relacionados ao conhecimento pedagógico, aspecto que se encontra frágil na formação desses profissionais.

Qualidades pessoais

As habilidades interpessoais foram identificadas no grupo dos preceptores, destacado na seguinte fala:

[...] tem que estar envolvido com a equipe, porque acaba que o estagiário, ele entra mas ele não conhece ninguém, então, acaba que a equipe também precisa saber que você é o responsável pelos pacientes e que você é o preceptor daquele estagiário...tem que ter esse envolvimento com a equipe, exemplo, nesse sentido de abordar tanto o paciente, mas também abordar a equipe também, né, eu acho que a

\footnotetext{
${ }^{1}$ Mestre em Ensino na Saúde pela Universidade Federal de Goiás, Goiânia, Brasil. E-mail: nutricionistafabricio@gmail.com.br ${ }^{2}$ Doutora em Ciências pela Universidade Federal de São Paulo, São Paulo, Brasil. Professora da Universidade Federal de Goiás, Goiânia. E-mail: idahelenamenezes@gmail.com

${ }^{3}$ Doutora em Medicina (Pediatria) pela Universidade Federal de São Paulo, São Paulo, Brasil. Professora da Universidade Federal de Goiás, Goiânia. E-mail: anaghettini@gmail.com
} 


\section{Programa de Pós-Graduação \\ em Ensino na Saúde - MEPES/UFG \\ DOSSIÊ \\ ENSINO NA SAÚDE}

V.17, N.01, 2021. ISSN: 1807-9342

forma de conduzir, a forma de estar lá com todo mundo, acho que tudo isso é modelo [...] (P5)

Rocha, Warmling e Toassi (2016) ao desenvolver um artigo que envolvet entrevistar entre preceptores e estudantes da área de odontologia apontaram a importância do preceptor apresentar algumas característica, tais como: empatia com as pessoas com que convive no ambiente de trabalho, facilidade de comunicação com os estudantes, na orientação, ensino, possibilitando trocas de conhecimento, experiências, discutindo casos clínicos, esclarecendo dúvidas, sendo paciente e cuidado com o processo de aprendizagem do estudante.

Ainda no grupo dos preceptores o atributo, Aberto, foi indicado em alguns discursos como uma qualidade pessoal que facilita o acesso do aluno ao preceptor.

[...] o primeiro é a comunicação, então, assim, eu acho que quando o preceptor de cara já se mostra uma pessoa aberta a conversar, discutir, isso já facilita, porque, facilita o acesso. (P3)

Em estudo realizado por Myrick e Youge (2004) na área de enfermagem, foi identificado que a comunicação entre preceptor e estudante é fundamental para o sucesso na experiência de preceptoria. Manter a comunicação aberta é o caminho para o bom andamento do processo. Estar disponível para atender o estudante pressupõe uma adequada forma de demonstrar disponibilidade.

Os preceptores são educadores, no sentido Freireano, que têm como objetivo construir junto com o estudante uma nova prática, que com base nos conhecimentos específicos de cada área possa atender aos problemas de saúde da população (SUCUPIRA; PEREIRA, 2004). O diálogo é importante na relação preceptor aluno, pois a troca de experiências acontece quando o estudante encontra um preceptor disposto a conversar e discutir.

Segundo Franco, Montes e Silva (2013), os estudantes apresentam notório desejo de

\footnotetext{
${ }^{1}$ Mestre em Ensino na Saúde pela Universidade Federal de Goiás, Goiânia, Brasil. E-mail: nutricionistafabricio@gmail.com.br

${ }^{2}$ Doutora em Ciências pela Universidade Federal de São Paulo, São Paulo, Brasil. Professora da Universidade Federal de Goiás, Goiânia. E-mail: idahelenamenezes@gmail.com

${ }^{3}$ Doutora em Medicina (Pediatria) pela Universidade Federal de São Paulo, São Paulo, Brasil. Professora da Universidade Federal de Goiás, Goiânia. E-mail: anaghettini@gmail.com
} 


\section{Programa de Pós-Graduação \\ em Ensino na Saúde - MEPES/UFG \\ DOSSIÊ \\ ENSINO NA SAÚDE}

executar o aprendizado prático junto com o preceptor. A segurança pela proximidade, a disponibilidade e paciência para esclarecimento de dúvidas, o apoio na realização de procedimentos e atividades clínicas são essenciais para o processo de aprendizado.

No grupo dos estudantes foi indicado outro atributo essencial para o preceptor: Ter ética.

[...] ética profissional que seria o principal, em todos os sentidos... na hora da elaboração de conduta [...] (A3)

O preceptor é o responsável pelo preparo profissional, ético e humanista dos estudantes, portanto aspectos éticos e morais são atributos essenciais a serem desenvolvidos por um bom preceptor (SKARE, 2012). A ética é demonstrada na fala destacada como atributo principal, já que o preceptor exerce influência na formação destes novos profissionais.

Em texto de Birden e colaboradores (2013), esses autores afirmam que o profissionalismo é aprendido de forma mais eficaz por meio da influência de educadores (modelos) que os estudantes encontram durante seu processo de formação. O preceptor, em alguns casos, pode ser o único profissional da prática que o aluno de graduação observará. Portanto, o exemplo (negativo ou positivo) para o estudante na rotina de trabalho no estágio será relevante para a formação da sua identidade profissional.

Qualidades profissionais

Segundo o discurso de um preceptor, gostar de fazer assistência é um atributo que o preceptor deve possuir, além do ensinar.

[...] O preceptor tem que ser alguém que goste de fazer assistência. [...] Assistência ao paciente? [...] Ele tem que gostar de ensino e de assistência. [...] porque o

\footnotetext{
${ }^{1}$ Mestre em Ensino na Saúde pela Universidade Federal de Goiás, Goiânia, Brasil. E-mail: nutricionistafabricio@gmail.com.br

${ }^{2}$ Doutora em Ciências pela Universidade Federal de São Paulo, São Paulo, Brasil. Professora da Universidade Federal de Goiás, Goiânia. E-mail: idahelenamenezes@gmail.com

${ }^{3}$ Doutora em Medicina (Pediatria) pela Universidade Federal de São Paulo, São Paulo, Brasil. Professora da Universidade Federal de Goiás, Goiânia. E-mail: anaghettini@gmail.com
} 


\section{Programa de Pós-Graduação

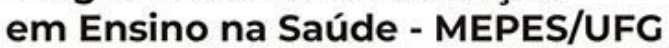 \\ DOSSIÊ \\ ENSINO NA SAÚDE}

V.17, N.01, 2021. ISSN: 1807-9342

professor, ele pode ser que goste só de ensino, está tudo certo [...] mas acho que o preceptor, ele precisa ter os dois. (P1)

É esperado que o preceptor ofereça ensino clínico diário aos estudantes durante o contato com as expectativas das práticas clínicas (BURNS, 2006). A prática é eixo estruturante no desenvolvimento do preceptor, dimensão que se articula com as experiências do profissional. Representa compreender o conhecimento como processo de construção, contextualizando em tempo e espaço estabelecidos (BATISTA, 2005). Assim o preceptor deve gostar do ensino e também da prática profissional (assistência ao paciente) devido ao seu papel junto ao estudante no campo de estágio.

Outro atributo identificado pelo grupo dos preceptores foi a Prática do cuidado humanizado ao paciente.

[...] Outra coisa assim que eu acho que eles veem muito é como a gente trata o paciente, o quanto a gente leva aquilo a sério, né, então, a empatia que a gente cria com o paciente, eles veem, assim, o quanto você trata o paciente da forma humanizada $[\ldots](\mathrm{P} 3)$

Segundo Chernicharo, Silva e Ferreira (2014), na área de enfermagem, a humanização na assistência foi caracterizada como uma prática de todos os profissionais. Essa deve ser embasada em uma relação entre profissional/paciente, por incluir características pessoais, por olhar para as necessidades individuais, pelo diálogo, escuta ativa, visão holística, empatia, valores morais e éticos, e inclui questões subjetivas tais como: amor, pensamento, valorização do ser, estabelecimento de vínculo, dentre outros.

O atributo do preceptor ter boa qualificação é exposto através da seguinte fala, pelos estudantes:

Os atributos essenciais é ter tido uma boa formação, e depois dela ter buscado

\footnotetext{
${ }^{1}$ Mestre em Ensino na Saúde pela Universidade Federal de Goiás, Goiânia, Brasil. E-mail: nutricionistafabricio@gmail.com.br

${ }^{2}$ Doutora em Ciências pela Universidade Federal de São Paulo, São Paulo, Brasil. Professora da Universidade Federal de Goiás, Goiânia. E-mail: idahelenamenezes@gmail.com

${ }^{3}$ Doutora em Medicina (Pediatria) pela Universidade Federal de São Paulo, São Paulo, Brasil. Professora da Universidade Federal de Goiás, Goiânia. E-mail: anaghettini@gmail.com
} 


\section{Programa de Pós-Graduação \\ em Ensino na Saúde - MEPES/UFG \\ DOSSIÊ \\ ENSINO NA SAÚDE}

V.17, N.01, 2021. ISSN: 1807-9342

aperfeiçoamento ou aprofundamento dessa formação, por meio de uma pós-graduação, mestrado, residência [...] (A7)

Quanto a formação profissional, todas preceptoras entrevistadas cursaram ou estavam cursando alguma pós-graduação: Duas com doutorado em andamento (17\%), duas com mestrado concluído (17\%), três com mestrado em andamento (25\%) e cinco com especialização concluída (21\%). Pode-se verificar com esses resultados que o grupo tem buscado a capacitação na área profissional e consequentemente na área do ensino.

Nas falas também emergiu o atributo do preceptor experiente destacado dentro das qualidades profissionais como atributo obrigatório. A vivência da prática é citada como uma forma do preceptor ser identificado como um Role Model. Na história da educação médica pode-se notar a figura de um profissional mais experiente, que se identifica com o papel do preceptor, que auxilia na formação profissional (BOTTI; REGO, 2011).

[...] tem que ter é experiência [...] tem que ter uma vivência prática para chegar a ser um preceptor modelo [...] (A7)

Nessa pesquisa o tempo de atuação de oito (66\%) das nutricionistas entrevistadas nos hospitais da pesquisa foram predominantemente de dois a três anos, indicando assim pouco de tempo de experiência no exercício da preceptoria e nove (75\%) tinham seis a sete anos de formadas.

\subsection{Atributos que afastam do modelo}

Nesse eixo surgiram várias subcategorias semelhantes as subcategorias que emergiram no eixo temático Atributos que aproximam do Modelo, são elas: Descompromisso com o processo de ensino-aprendizagem, Mau relacionamento, Falta de ética, Falta de qualificação e

\footnotetext{
${ }^{1}$ Mestre em Ensino na Saúde pela Universidade Federal de Goiás, Goiânia, Brasil. E-mail: nutricionistafabricio@gmail.com.br

${ }^{2}$ Doutora em Ciências pela Universidade Federal de São Paulo, São Paulo, Brasil. Professora da Universidade Federal de Goiás, Goiânia. E-mail: idahelenamenezes@gmail.com

${ }^{3}$ Doutora em Medicina (Pediatria) pela Universidade Federal de São Paulo, São Paulo, Brasil. Professora da Universidade Federal de Goiás, Goiânia. E-mail: anaghettini@gmail.com
} 
Não realiza um tratamento humanizado. No entanto será abordado neste eixo apenas as subcategorias inéditas.

Dificuldades Relacionadas ao Ensino

Nesta categoria emergiu a seguinte subcategoria no grupo dos estudantes: Desinteresse pela preceptoria:

[...] aqueles que realmente fazem pouco caso, que acontece de vez em quando [...] com o estagiário, não dá atenção, não faz questão, acho que é ruim [...] (A13)

O preceptor deve entender que dentre as funções do preceptor no campo de estágio estão: acompanhar, conduzir e intervir durante o processo de ensino aprendizado dos estudantes. No entanto, de acordo com Oliveira e Petta (2014) na realidade atual, os preceptores de graduação ou pós-graduação em exercício são, em geral, profissionais com especialização em cursos de saúde, formados no modelo biomédico e num currículo tradicional, sem capacitação na área da educação e em cursos que não preparam o indivíduo para a docência (FAJARDO; CECCIM, 2010).

\section{Dificuldades Profissionais}

No grupo dos preceptores emergiu a seguinte subcategoria: Falta de maturidade como visto na fala do preceptor abaixo:

\footnotetext{
[...] dificuldade com hierarquias. [...] insubmissão [...] o preceptor, ele tem que ser uma pessoa madura, aí, eu não sei te falar com clareza se a maturidade está associada com a idade, mas, por exemplo, se eu for me comparar há 10 anos atrás ou quando eu ingressei no serviço público é muito diferente [...] é uma questão de amadurecimento mesmo né. $E$ às vezes essa pouca idade (do estudante) se confunde com a pouca idade do preceptor, então, acaba que fica um pouco confuso[...] (P2)
}

\footnotetext{
${ }^{1}$ Mestre em Ensino na Saúde pela Universidade Federal de Goiás, Goiânia, Brasil. E-mail: nutricionistafabricio@gmail.com.br ${ }^{2}$ Doutora em Ciências pela Universidade Federal de São Paulo, São Paulo, Brasil. Professora da Universidade Federal de Goiás, Goiânia. E-mail: idahelenamenezes@gmail.com

${ }^{3}$ Doutora em Medicina (Pediatria) pela Universidade Federal de São Paulo, São Paulo, Brasil. Professora da Universidade Federal de Goiás, Goiânia. E-mail: anaghettini@gmail.com
} 


\section{Programa de Pós-Graduação \\ em Ensino na Saúde - MEPES/UFG \\ DOSSIÊ \\ ENSINO NA SAÚDE}

V.17, N.01, 2021. ISSN: 1807-9342

De acordo com o preceptor, o tempo pode trazer um certo amadurecimento no relacionamento entre todos atores envolvidos no serviço da preceptoria: estudantes, gestores locais e professores das instituições de ensino. Wuillaume e colaboradores (2000), na área de preceptoria médica, identificaram alguns atributos para o bom preceptor, tais como: conhecimento; conhecimento científico; conhecimento teórico; conhecimento técnico; experiência; experiência clínica; vivência prática; prática médica. Dentre esses atributos foram identificados alguns que estão relacionados com o tempo, portanto a vivência em campo de prática pode ajudar no relacionamento entre professor e aluno.

Por fim, emergiu do grupo de estudantes a subcategoria: Não sabe trabalhar em equipe.

[...] Que não conseguem trabalhar em equipe também, que são mais distantes, até mesmo dos outros profissionais da área hospitalar [...] (A13)

Os preceptores devem ser capazes de realizar atividades com profissionais de outras áreas. O trabalho em equipe precisa ser articulado, pois é de grande importância como cada profissão tem uma visão entre setores, a qual abrange além de uma área profissional (MARINS, 2011). Na fala do estudante o preceptor que não consegue trabalhar em equipe ou é distante dos outros profissionais que trabalham no ambiente hospitalar não é considerado um modelo de preceptor.

Dificuldades Pessoais

Nesta categoria não emergiu subcategorias inéditas.

\section{CONSIDERAÇÕES FINAIS}

\footnotetext{
${ }^{1}$ Mestre em Ensino na Saúde pela Universidade Federal de Goiás, Goiânia, Brasil. E-mail: nutricionistafabricio@gmail.com.br ${ }^{2}$ Doutora em Ciências pela Universidade Federal de São Paulo, São Paulo, Brasil. Professora da Universidade Federal de Goiás, Goiânia. E-mail: idahelenamenezes@gmail.com

${ }^{3}$ Doutora em Medicina (Pediatria) pela Universidade Federal de São Paulo, São Paulo, Brasil. Professora da Universidade Federal de Goiás, Goiânia. E-mail: anaghettini@gmail.com
} 


\section{Programa de Pós-Graduação \\ em Ensino na Saúde - MEPES/UFG \\ DOSSIÊ \\ ENSINO NA SAÚDE}

Nessa pesquisa o tema central das subcategorias que emergiram foi a relação preceptor-aluno, surgindo poucas vezes a figura do paciente. Pode ser que os entrevistados não visualizaram ou não perceberam a importância desta relação na formação da identidade do futuro profissional.

Comparando os resultados entre os dois grupos verificou-se que surgiram várias categorias e subcategorias semelhantes sugerindo que a visão do preceptor como modelo é percebida de forma similar. No entanto emergiram mais subcategorias no grupo dos estudantes que no grupo dos preceptores, o que pode demonstrar a habilidade dos estudantes em ver de maneira mais ampla o papel do preceptor.

Além disso, em relação ao conceito de preceptor de nutrição clínica os dois grupos identificaram o Preceptor como um profissional que ensina tanto a prática como a teoria e que cuida do estudante. No entanto apenas o grupo dos estudantes conseguiram visualizar o papel do preceptor como "Espelho" (Modelo). Talvez o grupo dos alunos consiga ver mais claramente o papel do preceptor como modelo no campo de estágio.

Assim, é necessário que seja realizado mais estudos aprofundados sobre os atributos do preceptor modelo de nutrição clínica e encontre-se novas estratégias para a formação da identidade profissional dos novos profissionais.

\section{REFERÊNCIAS}

AUTONOMO, F.R.O.M. et al. A preceptoria na formação médica e multiprofissional com ênfase na atenção primária - Análise das publicações brasileiras. Revista Brasileira de Educação Médica. Rio de Janeiro, v.39, n.2, p.316-327, 2015.

BATISTA, N.A. Desenvolvimento docente na área da saúde: Uma análise. Revista Trabalho, Educação e Saúde. Rio de Janeiro, v.3, n.2, p.283-294, 2005.

BARDIN, L. Análise de Conteúdo. 1ª ed, São Paulo: Edições 70, 2016, p. 279.

\footnotetext{
${ }^{1}$ Mestre em Ensino na Saúde pela Universidade Federal de Goiás, Goiânia, Brasil. E-mail: nutricionistafabricio@gmail.com.br

${ }^{2}$ Doutora em Ciências pela Universidade Federal de São Paulo, São Paulo, Brasil. Professora da Universidade Federal de Goiás, Goiânia. E-mail: idahelenamenezes@gmail.com

${ }^{3}$ Doutora em Medicina (Pediatria) pela Universidade Federal de São Paulo, São Paulo, Brasil. Professora da Universidade Federal de Goiás, Goiânia. E-mail: anaghettini@gmail.com
} 


\section{Programa de Pós-Graduação \\ em Ensino na Saúde - MEPES/UFG \\ DOSSIÊ \\ ENSINO NA SAÚDE}

V.17, N.01, 2021. ISSN: 1807-9342

BANDURA, A.; AZZI, R.G.; POLYDORO, S. Teoria Social Cognitiva: Conceitos Básicos. Porto Alegre: Artmed, 2008, p. 176.

BERNAT, J.L. Restoring medical professionalism. Neurolology. v.79, n.8. p.820-827, 2012.

BIRDEN, H., et al. Teachig professionalism in medial education: A Best Evidence Medical Education(BEME) systematic review. Medical Teacher. v.35. p.1252-1266, 2013.

BOTTI, S.H.O.; REGO, S.T.A. Docente_clínico: o complexo papel do preceptor na residência médica. Physis Revista de Saúde Coletiva; Rio de Janeiro, v.21, n.1, p.65-85, 2011.

BOTTI, S.H.O.; REGO, S. Preceptor, Supervisor, Tutor e Mentor: Quais são seus papéis? Revista Brasileira de Educação Médica; Rio de Janeiro, v.32, n.3, p.363-373, 2008.

BRASIL. Conselho Nacional de Educação. Câmara de Educação Superior. Resolução n. 5 de 07 de novembro de 2001. Institui Diretrizes Curriculares Nacionais do Curso de Graduação em Nutrição. Brasília. Disponível em: <

http://portal.mec.gov.br/cne/arquivos/pdf/CES05.pdf>. Acesso em: 12 mai. 2018.

Ministério. Ministério da Saúde. Portaria nº 1.111 de 05 de julho de 2005. Fixa

normas para implementação e a execução do Programa de Bolsas para a Educação pelo trabalho. Brasília. Disponível em:

$<$ http://bvsms.saude.gov.br/bvs/saudelegis/gm/2005/prt1111_05_07_2005.html >. Acesso em: 30 mar. 2018.

Resolução 466 de 12 de dezembro de 2012. Diretrizes e normas regulamentadoras de pesquisas envolvendo seres humanos. Ministério da Saúde/Conselho Nacional de Saúde, Brasília, 12 de dezembro de 2. Disponível em: <

http://conselho.saude.gov.br/resolucoes/2012/Reso466.pdf>. Acesso em: 18 de jun. 2016.

BORGES, M.C et al. Avaliação formativa e feedback como ferramenta de aprendizado na formação de profissionais da saúde. Revista Medicina. Ribeirão Preto, v.47, n.3. p.324-331, 2014.

BURNS, C. et al. Mastering the preceptor role: challenges of clinical teaching. Journal of Pediatric Health Care. Nova Iorque, v.20, n.3. p.172-183, may-jun, 2006.

\footnotetext{
${ }^{1}$ Mestre em Ensino na Saúde pela Universidade Federal de Goiás, Goiânia, Brasil. E-mail: nutricionistafabricio@gmail.com.br

${ }^{2}$ Doutora em Ciências pela Universidade Federal de São Paulo, São Paulo, Brasil. Professora da Universidade Federal de Goiás, Goiânia. E-mail: idahelenamenezes@gmail.com

${ }^{3}$ Doutora em Medicina (Pediatria) pela Universidade Federal de São Paulo, São Paulo, Brasil. Professora da Universidade Federal de Goiás, Goiânia. E-mail: anaghettini@gmail.com
} 


\section{Programa de Pós-Graduação \\ em Ensino na Saúde - MEPES/UFG \\ DOSSIÊ \\ ENSINO NA SAÚDE}

CASSOLA, T. P. et al. Percepção de tutores, preceptores e bolsistas em relação à sua inserção no programa de educação pelo trabalho em saúde. Disciplinarum Scientia. Série: Ciências da Saúde. Santa Maria, v.15, n.2, p.171-179, 2014.

CERQUEIRA, P. A formação pedagógica de preceptores dos estudantes da área da saúde: uma conversa em três tempos. In: RIBEIRO, V.M.B. (org). Formação pedagógica de preceptores do ensino na saúde. Juiz de Fora: Ed. UFJF, p. 125, 2011.

CHERNICHARO, I.M.; SILVA, F.D.; FERREIRA, M.A. Caracterização do termo humanização na assistência por profissionais de enfermagem. Escola Anna Nery Revista de Enfermagem; Rio de Janeiro, v.18, n.1, p.156-162, jan/mar. 2014.

CONSELHO FEDERAL DE NUTRICIONISTAS. Resolução CFN nº 418/2008. Dispõe sobre a responsabilidade do nutricionista quanto às atividades desenvolvidas por estagiários de nutrição e dá outras providências. Disponível em: http://cfn.org.br/novosite/pdf/res418.pdf. Acesso em: 11 mar. 2018.

FAJARDO, A.P.; CECCIM, R.B. O trabalho da preceptoria nos tempos de residência em área profissional da saúde. In: FAJARDO, A.P.; ROCHA, C.M.F.; PASINI, V.L. (Org.).

Residência em saúde: fazeres e saberes na formação em saúde. Porto Alegre: Ed. Hospital Nossa Senhora da Conceição S.A., p. 260, 2010.

FEUERWERKER, L.C.M. As identidades do preceptor: assistência, ensino e orientação. In: RIBEIRO, V.M.B. (org). Formação pedagógica de preceptores do ensino na saúde. Juiz de Fora: Ed. UFJF, p. 125, 2011.

FRANCO, F.M.; MONTES, M.A.A.; SILVA, A.R. Visão discente do papel da preceptoria médica na formação dos alunos de medicina. Alexandria - Revista de Educação em Ciência e Tecnologia; Florianópolis, v.6, n.2, p.229-249, Jun., 2013.

FRANKLIN, N. Clinical supervision in undergraduate nursing students: A review of the literature. e-Journal of Business Education e Scholaship of Teaching. v.7, n. 1, p.34-42, 2013.

HEALY, A.N. et al. Role Models and Mentors in Sugery. The American Journal of Sugery, Birminghan, v.204, n.2, p.256-261, agosto, 2012.

\footnotetext{
${ }^{1}$ Mestre em Ensino na Saúde pela Universidade Federal de Goiás, Goiânia, Brasil. E-mail: nutricionistafabricio@gmail.com.br

${ }^{2}$ Doutora em Ciências pela Universidade Federal de São Paulo, São Paulo, Brasil. Professora da Universidade Federal de Goiás, Goiânia. E-mail: idahelenamenezes@gmail.com

${ }^{3}$ Doutora em Medicina (Pediatria) pela Universidade Federal de São Paulo, São Paulo, Brasil. Professora da Universidade Federal de Goiás, Goiânia. E-mail: anaghettini@gmail.com
} 


\section{Programa de Pós-Graduação \\ em Ensino na Saúde - MEPES/UFG \\ DOSSIÊ \\ ENSINO NA SAÚDE}

LEEUW, J. et al. The Attributes of the Clinical Trainer as a Role Model: A Systematic Review. Academic Medicine. Amsterdam, v.88, n.1, p.26-34, 2013.

LIE, D. A Framework for Enhancing and Assessing Cultural Competency Training. The Kaohsiung Journal of Medical Sciences. v.25, n.9, p.486-492, 2009.

LIMA, P.A.B.; ROZENDO, C.A. Desafios e possibilidades no exercício da preceptoria do Pró-PET-Saúde. Interface - Comunicação Saúde Educação. Botucatu, v.19, supl.1, p.779-791p, 2015.

KENNY, N.P.; MANN, K.V.; MACLEOD, H. Role modeling in physicians professional formation: Reconsidering an essencial but untapped education strategy. Academic Medicine. v.78, n.12, p.1203-1210, 2003.

MACLELLAN, D.L.; LORDLY, D. The socialization of Dietetic Students: Influence of the Preceptor Role. Journal of Allied Health. Washington, v.37, n.2. p. 81-92, 2008.

MARINS, J.J.N. Formação de preceptores para área da saúde. In: RIBEIRO, V.M.B. (org). Formação pedagógica de preceptores do ensino na saúde. Juiz de Fora: Ed. UFJF, 2011. $125 \mathrm{p}$.

MINAYO, M.C.S. (Org) et.al. Pesquisa Social: teoria, método e criatividade. $22^{\mathrm{a}}$ ed., Petrópolis: Vozes, 2003.

MISSAKA, H.; RIBEIRO, V.M.B. A preceptoria na formação médica: o que dizem os trabalhos nos congressos brasileiros de educação médica 2007-2009. Revista Brasileira de Educação Médica; Rio de Janeiro, v.35, n.3, p.303-310, 2011.

MOHR, A. A formação pedagógica dos profissionais da área da saúde. In: RIBEIRO, V.M.B. (org). Formação pedagógica de preceptores do ensino na saúde. Juiz de Fora: Ed. UFJF, 2011. 125p.

MONTEIRO, D.M.; LEHER, E.M.T.; RIBEIRO, V.M.B. Da educação continuada à educação permanente: a construção do modelo de formação pedagógica para preceptores de Internato Médico. In: RIBEIRO, V.M.B. (org). Formação pedagógica de preceptores do ensino na saúde. Juiz de Fora: Ed. UFJF, 2011. 125p.

\footnotetext{
${ }^{1}$ Mestre em Ensino na Saúde pela Universidade Federal de Goiás, Goiânia, Brasil. E-mail: nutricionistafabricio@gmail.com.br

${ }^{2}$ Doutora em Ciências pela Universidade Federal de São Paulo, São Paulo, Brasil. Professora da Universidade Federal de Goiás, Goiânia. E-mail: idahelenamenezes@gmail.com

${ }^{3}$ Doutora em Medicina (Pediatria) pela Universidade Federal de São Paulo, São Paulo, Brasil. Professora da Universidade Federal de Goiás, Goiânia. E-mail: anaghettini@gmail.com
} 


\section{Programa de Pós-Graduação \\ em Ensino na Saúde - MEPES/UFG \\ DOSSIÊ \\ ENSINO NA SAÚDE}

MOSSOP, L.H. Is it Time to Define Veterinary Professionalism. Journal of Veterinary Medical Education. v. 39, n.1, p.93-100, 2012.

MYRICK, F.; YONGE.O. Nursing Preceptorship: connecting practice and education. Edmont: University of Alberta, 2004. 224p.

PAICE, E.; HEARD, S.; MOSS, F. How important are role models in making good doctors. British Medical Journal. Londres, v.325, n.28. p.707-710, 2002.

OLIVEIRA, M.S.; PETTA, H.L. Novas necessidades de formação para o SUS: educação na saúde para preceptores. In: OLIVEIRA, M.S. et al (org). Curso de especialização Educação na Saúde para Preceptores do SUS - Caderno do curso SUS. São Paulo: Instituto Sírio-Libanês de Ensino e Pesquisa - Ministério da Saúde, 2014. 48p.

RAMOS, M. Trabalho, educação e correntes pedagógicas no Brasil: um estudo a partir da formação dos trabalhadores técnicos da saúde. Rio de Janeiro. EPSJV-UFRJ, 2010, 290 p.

ROCHA, P.F.; WARMLING, C.M.; TOASSI, R.F.C. Preceptoria como modalidade de ensino na saúde: Atuação e carcterística do preceptor Cirurgião-Dentista na atenção primária. Revista Saberes Plurais - Educação na Saúde. Porto Alegre, v.1, n.1. p.96-112, 2016.

ROSSONI, E.; LAMPERT, J. Formação de profissionais para o Sistema único de saúde e as diretrizes curriculares. Boletim da Saúde. Porto Alegre, v.18, n.1, p.87-98, jan/jun, 2004.

ROYAL COLLEGE OF PHYSICIANS OF LONDON. Doctors in Society: Medical Professionalism in a Changing World (London, Royal College of Physicians of London) (2005).

SANTOS, S.C. O processo de ensino aprendizagem e a relação professor aluno: Aplicação dos "Sete princípios para a boa prática na educação de ensino superior". Cadernos de Pesquisas em Administração. São Paulo, v. 8, n.1, p.70-82, jan-mar, 2001.

SKARE, T.L. Metodologia do ensino na preceptoria da residência médica. Revista do Médico de Residente, Curitiba, v. 4, n.2, p.116-120, abr-jun, 2012.

SUCUPIRA, A. C. S. L.; PEREIRA A. A preceptoria na residência em saúde da Família. Sanare; Sobral, v. 5, n.1, p.47-53, 2004.

\footnotetext{
${ }^{1}$ Mestre em Ensino na Saúde pela Universidade Federal de Goiás, Goiânia, Brasil. E-mail: nutricionistafabricio@gmail.com.br

${ }^{2}$ Doutora em Ciências pela Universidade Federal de São Paulo, São Paulo, Brasil. Professora da Universidade Federal de Goiás, Goiânia. E-mail: idahelenamenezes@gmail.com

${ }^{3}$ Doutora em Medicina (Pediatria) pela Universidade Federal de São Paulo, São Paulo, Brasil. Professora da Universidade Federal de Goiás, Goiânia. E-mail: anaghettini@gmail.com
} 


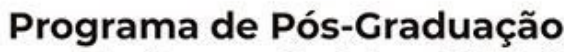

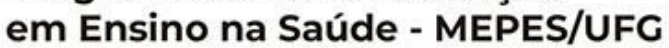 \\ DOSSIÊ \\ ENSINO NA SAÚDE}

V.17, N.01, 2021.

ISSN: 1807-9342

USHER, K. et. al. An exploration of the preceptor role: preceptors' perceptions of benefits, rewards, supports and commitment to the preceptor role. Journal of Advanced Nursing. v.29, n.2, p.560-514, 1999.

WRIGHT, S.M. et. al. Attributes of excellent attending-physician role models. The New England Journal of Medicine. v.339, n.27, p.1986-1993, 1998.

WUILLAUME, S.M.; BATISTA, N.A. O Preceptor na residência médica em Pediatria: principais atributos. Jornal de Pediatria. Rio de Janeiro, v.76, n.5, p.333-338, 2000.

YOUNG, S. Factors associated with students' perception of preceptor excellence. American Journal of Pharmaceutical Education. v.78, n.3, p.1-6, 2014.

\footnotetext{
${ }^{1}$ Mestre em Ensino na Saúde pela Universidade Federal de Goiás, Goiânia, Brasil. E-mail: nutricionistafabricio@gmail.com.br ${ }^{2}$ Doutora em Ciências pela Universidade Federal de São Paulo, São Paulo, Brasil. Professora da Universidade Federal de Goiás, Goiânia. E-mail: idahelenamenezes@gmail.com

${ }^{3}$ Doutora em Medicina (Pediatria) pela Universidade Federal de São Paulo, São Paulo, Brasil. Professora da Universidade Federal de Goiás, Goiânia. E-mail: anaghettini@gmail.com
} 